\title{
Electrochemical Amperometric Biosensors Based on Directly Synthesized Carbon Nanotube Electrodes
}

\author{
Kenzo Maehashi* and Kazuhiko Matsumoto \\ The Institute of Scientific and Industrial Research, Osaka University, \\ 8-1 Mihogaoka, Ibaraki, Osaka 567-0047, Japan
}

(Received February 25, 2009; accepted June 8, 2009)

Key words: label-free biosensors, carbon nanotube electrodes, immunosensors, microfludic chips, pneumatic micropumps, cancer markers

In recent years, label-free detections of biomolecules have attracted great attention in many fields of life science such as genomics, clinical diagnosis, and practical pharmacy. In this article, we review electrochemical amperometric biosensors based on carbon nanotube (CNT) electrodes. In electrochemical detections, CNT electrodes promote electron transfer reactions on CNT surfaces. Since CNTs have a large specific surface area, the direct synthesization of CNTs on electrodes in amperometric biosensors is expected to significantly enhance electroactive surface area. In this review, we discuss the technology and performance of the electrochemical biosensors based on CNT electrodes and describe microfludic chips with multibiosensors based on CNT electrodes for commercialization.

\section{Introduction}

Biosensors for the detection of biomolecules such as DNA and proteins have attracted great attention in many areas of life science such as practical pharmacy, genomics, and clinical diagnosis for health care. ${ }^{(1-4)}$ In particular, label-free monitoring of biorecognition events provides a promising platform, which is simpler, less expensive, and requires less energy. Thus, it is very suitable for minimization of biosensors. Rapid testing of different proteins is also required in various applications.

Electrochemical amperometric biosensors are one of the promising tools for labelfree monitoring of biomolecules. ${ }^{(5)}$ In electrochemical amperometric sensing, in general, three types of electrode are arranged: working, reference, and counter electrodes. On the

"Corresponding author: e-mail: maehashi@sanken.osaka-u.ac.jp 
working-electrode surfaces, electroactive biomolecules are oxidized or reduced, and then electron transfer reactions occur. Therefore, choosing appropriate materials of working electrodes is fundamental to the success of electrochemical measurements. Recently, gold, platinum, and glassy carbon have been utilized as working electrodes. However, such conventional working electrodes do not have sufficient sensitivity when they are downsized to the micrometer size for hand-held multibiosensors.

Carbon nanotubes (CNTs) are one of the most promising materials for the development of nanodevices, owing to their unique electrical and mechanical properties and nanoscale size. ${ }^{(6-8)}$ CNTs are also utilized as a channel of CNT field-effect transistors (CNTFETs) for label-free potentiometric biosensors. ${ }^{(9-11)}$ Biomolecules such as DNA and proteins were detected using CNTFETs. ${ }^{(12-18)}$ CNTs are also reported to have a higher ability to promote electron transfer reactions than conventional metal electrodes for electrochemical measurements. ${ }^{(19)}$ When CNTs have a high aspect ratio, the total surface area of working electrodes becomes larger when CNTs are modified on the surface of electrodes. As a result, highly sensitive detection of biomolecules is expected using CNT-modified electrodes for electrochemical analysis. Recently, CNT-modified electrodes have been fabricated by scattering CNTs on the metal surfaces. ${ }^{(20-22)}$ However, they are considered to have high contact resistance between CNTs and metal electrodes. Moreover, it seems difficult to integrate the working electrodes using this method.

In this article, we review electrochemical amperometric biosensors based on CNTmodified electrodes, with special reference to related works carried out in our laboratory. Since CNTs are directly synthesized on the surface of bare metal electrodes, the total surface area of working electrodes is estimated to be approximately a thousand times larger on the same site than that of a bare metal electrode, as shown in Fig. 1. As a result, the sensitivity in detection of biomolecules will be improved and the CNT working electrodes can be integrated into multibiosensors. In this review, we focus on the technology, characteristics, and developments for commercialization of electrochemical amperometric biosensors based on CNT-modified electrodes.

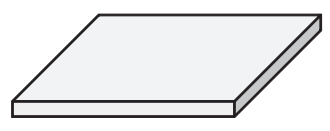

(a)

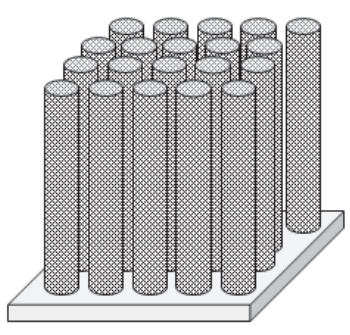

(b)

Fig. 1. Schematic illustration of working electrodes in electrochemical detection: (a) conventional metal and (b) CNT electrodes. 


\section{Biosensors Based on CNT Electrodes}

The development of CNT-arrayed electrode chips is reviewed in this section. CNTarrayed electrodes were directly synthesized on Pt surfaces by thermal chemical vapor deposition, ${ }^{(23)}$ and were also arrayed on a chip, ${ }^{(24)}$ as shown in Fig. 2(a). Figure 2(b) shows an optical microscope image of the three-electrode system that was applied in electrochemical measurements. CNT-arrayed electrodes, a Pt wire, and $\mathrm{Ag} / \mathrm{AgCl}$ were used as the working, counter, and reference electrodes, respectively. The working electrodes were surrounded by a silicone chamber attached to the substrate, as shown in Fig. 2(b). The electrochemical characteristics of the devices were investigated using $\mathrm{K}_{3}\left[\mathrm{Fe}(\mathrm{CN})_{6}\right]$ and electroactive amino acids: L-tyrosine, L-cysteine, and L-tryptophan. A low oxidation potential for amino acids was obtained compared with Pt disc electrodes. ${ }^{(25)}$ The electrochemical measurements using $\mathrm{K}_{3}\left[\mathrm{Fe}(\mathrm{CN})_{6}\right]$ and amino acids revealed that the peak current intensities using the CNT-arrayed electrodes were about 100-fold higher than those using bare Pt electrodes. The results indicated that the biosensors based on CNT-arrayed electrodes showed a high sensitivity suitable to detect biomolecules.

Next, to selectively detect a cancer marker, prostate-specific antigen (PSA), using the CNT electrodes, monoclonal antibodies against prostate-specific antigen (PSA-mAb) were covalently anchored onto the CNTs using 1-pyrenebutanoic acid succinimidyl ester

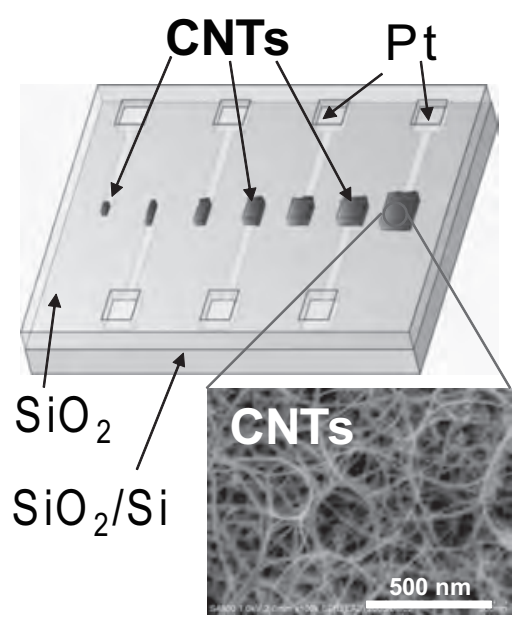

(a)

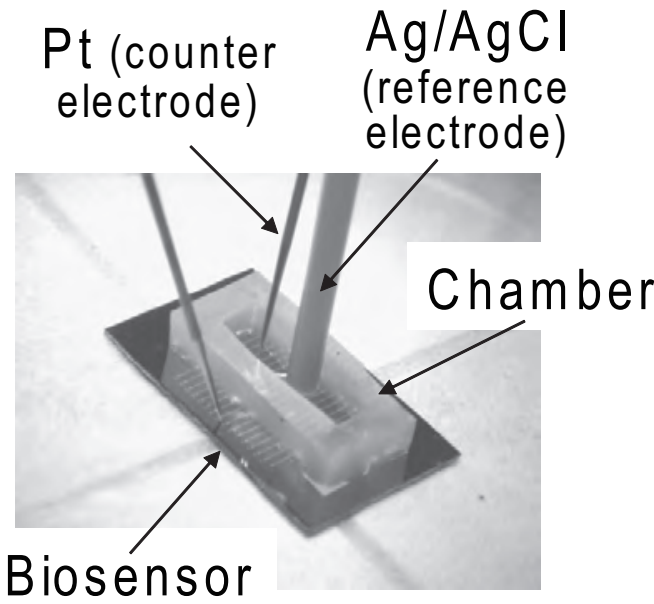

(b)

Fig. 2. (a) Schematic illustration of electrochemical biosensor based on CNT-arrayed electrodes. A scanning electron microscopy (SEM) image of a CNT electrode is shown in the inset. (b) Optical image of experimental setup of electrochemical biosensor based on CNT-arrayed electrodes. 
(Linker), as shown in Fig. 3(a). Figure 3(b) shows the electrochemical signals of CNTmodified electrodes recorded using differential pulse voltammetry (DPV). ${ }^{(26)}$ The peak current signal was obtained at $+0.5 \mathrm{~V}$ from only PSA-mAb, as indicated by the dotted line in Fig. 3(b). After the introduction of $1 \mathrm{ng} / \mathrm{mL}$ PSA on the PSA-mAb-modified CNT electrodes, the electrochemical current signal significantly increased, as shown by the

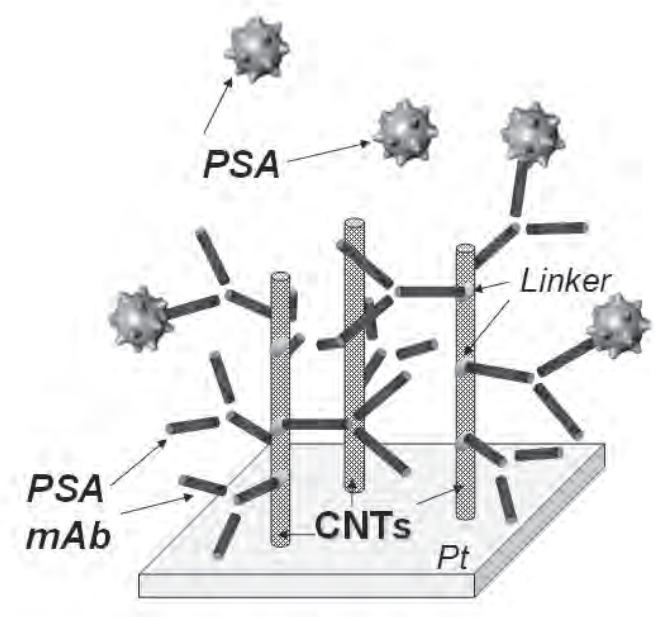

(a)

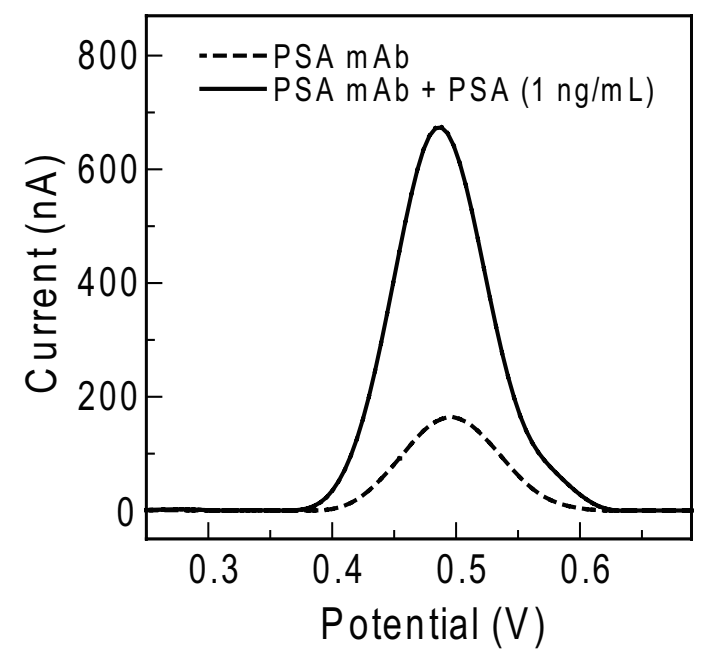

(b)

Fig. 3. (a) Schematic illustration of label-free electrochemical immunosensor. PSA-mAb was covalently immobilized on the CNTs using linkers. (b) Electrochemical signals of CNT-modified electrodes recorded using DPV. The dotted and solid lines correspond to the electrochemical signals from PSA-mAb and after introduction of $1 \mathrm{ng} / \mathrm{mL}$ PSA, respectivity. 
solid line in Fig. 3(b), indicating that the antigen-antibody complex was formed. The selectivity of the biosensor was also observed in a study using bovine serum albumin as the nontarget protein. PSA in the range of $0.25-1 \mathrm{ng} / \mathrm{mL}$ was effectively detected using the CNT electrodes. Since the cutoff limit of PSA for distinguishing between prostate hyperplasia and cancer is $4 \mathrm{ng} / \mathrm{mL}$, the performance of the label-free electrochemical immunosensor seems promising for further clinical applications. Furthermore, the nanoscale features by semiconductor processing made it possible to fabricate arrays with extremely high density and compatibility for further integration.

\section{Microfluidic Chips with Multibiosensors Based on CNT Electrodes}

In this section, microfluidic chips based on CNT electrodes are reviewed. The micrototal analysis system ( $\mu$ TAS) has attracted attention worldwide. ${ }^{(27-29)}$ In this system, the units of measurement are integrated using semiconducting fine processes, and all analytical processes are automatically carried out on one chip. It has the dual benefits of consumption of only a very small amount of reagents for analysis and of a markedly reduced analysis time. Significant research and development efforts have been devoted to producing microfluidic chips for the realization of $\mu \mathrm{TAS}$. Our group fabricated microfluidic chips using the combination of amperometric biosensors based on CNTarrayed electrodes and microchannels with pneumatic micropumps.

Figure 4(a) shows a schematic image of microfluidic chips based on CNT-arrayed electrodes. ${ }^{(30)}$ One chip has two liquid inlets, two pumps, and one liquid outlet. The pneumatic micropumps were composed of three poly(dimethylsiloxane) (PDMS) layers: an air layer, an intermediate membrane, and a liquid layer, as shown in Fig. 4(b). The experimental setup of a microfluidic chip is shown in Fig. 4(c). The air layers of the pneumatic micropumps were connected to the air pressure control. By pulling the air layers of the drive section, the reagents were sucked from inlets to the valve. Subsequently, by pushing them, the reagents were pushed out to the electrodes. By repeatedly pulling and pushing them, the reagents were constantly introduced to microchannels. The check valves prevented unexpected reverse flow and diffusion.

In the chip, phosphate buffer solution (PBS) and $\mathrm{K}_{3}\left[\mathrm{Fe}(\mathrm{CN})_{6}\right]$ were introduced into the CNT electrodes using two pneumatic micropumps. ${ }^{(30)}$ Electrochemical measurements indicated that the chips can automatically exchange reagents on the CNT electrodes and clearly detect molecules. Moreover, by modifying the CNT electrodes with the enzyme glucose oxidase, as shown in Fig. 5(a), glucose molecules were detected by chronoamperometry (CA). Figure 5(b) shows the time dependence of electrochemical signals determined by $\mathrm{CA}$ at an applied potential of $450 \mathrm{mV}$ vs $\mathrm{Ag} / \mathrm{AgCl}$. Glucose samples at three concentrations and PBS were alternately introduced into CNT electrodes from different inlets using two pumps. In Fig. 5(b), the amperometric response to glucose at each concentration is clearly observed. In addition, after PBS was introduced, the signals of glucose disappeared, indicating that PBS can rinse glucose out of the surfaces of the CNT electrodes. This result clearly revealed a linear response to glucose concentration within the range from 5 to $20 \mathrm{mg} / \mathrm{mL}$. These results indicate that glucose 


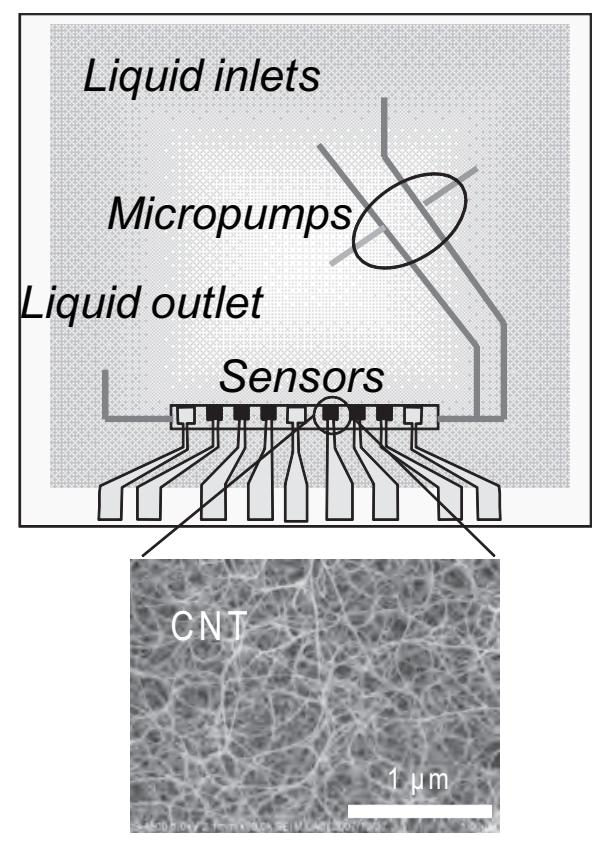

(a)

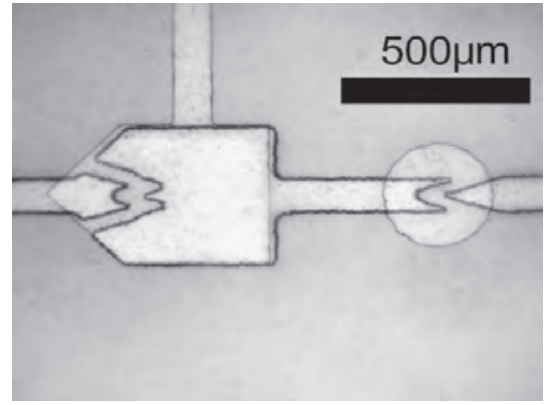

(b)

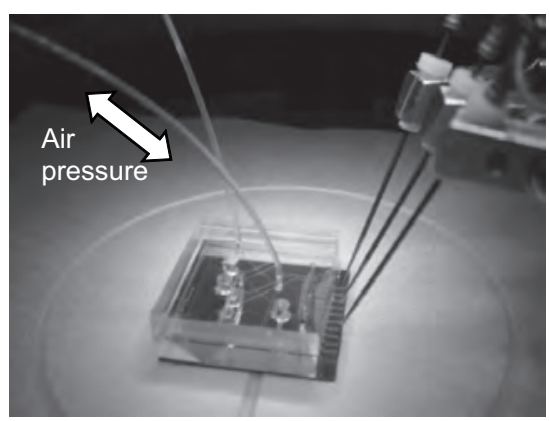

(c)

Fig. 4. (a) Schematic illustration of microfluidic chip based on CNT electrodes. A SEM image of a CNT electrode is shown in the inset. Optical image of (b) a pneumatic micropump and (c) experimental setup of microfluidic chip based on CNT electrodes.

can be quantified using the CNT electrodes modified with glucose oxidase, and PBS washes the glucose out of the CNT electrodes in the system.

Furthermore, for hand-held biochips, microfluidic multi-immunosensors were fabricated. $^{(31)}$ A schematic view of the microfluidic amperometric chip based on CNT electrodes is shown in Fig. 6(a). Four liquid inlets, six pumps, one liquid outlet, and three channels were integrated in the chip. Twelve CNT working electrodes, three Pt counter electrodes, and three $\mathrm{Ag} / \mathrm{AgCl}$ reference electrodes were also arrayed on the substrate, as shown in Fig. 6(b). In the microfluidic systems, four types of reagent were transported from each liquid inlet to arrayed microelectrodes using integrated pumps without unexpected reverse flow and diffusion. Simultaneous detection of two types of cancer marker, PSA and human chorionic gonadotropin (hCG), using the chip was reported. First, two types of antibody, PSA-mAb and hCG antibody, were automatically immobilized onto different CNT electrodes using the microfluidic systems. Next, PSA and hCG were injected into corresponding CNT electrodes. Finally, after rinsing all electrodes with blank PBS, PSA and hCG were simultaneously detected by DPV. 


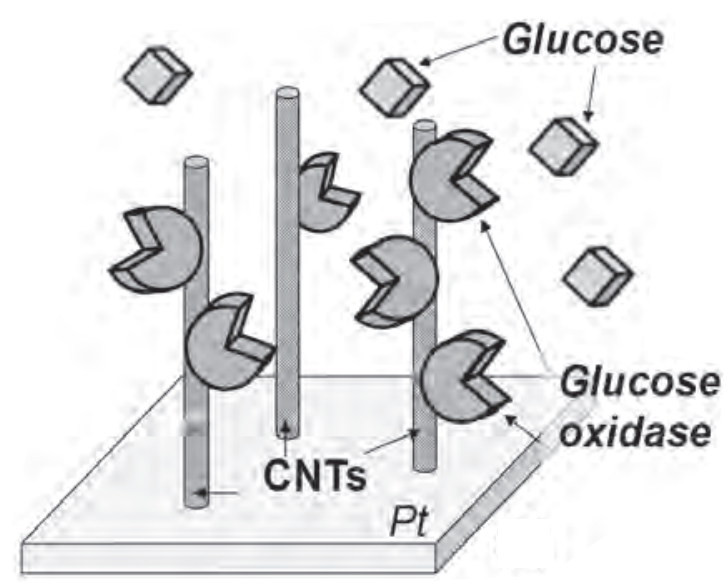

(a)

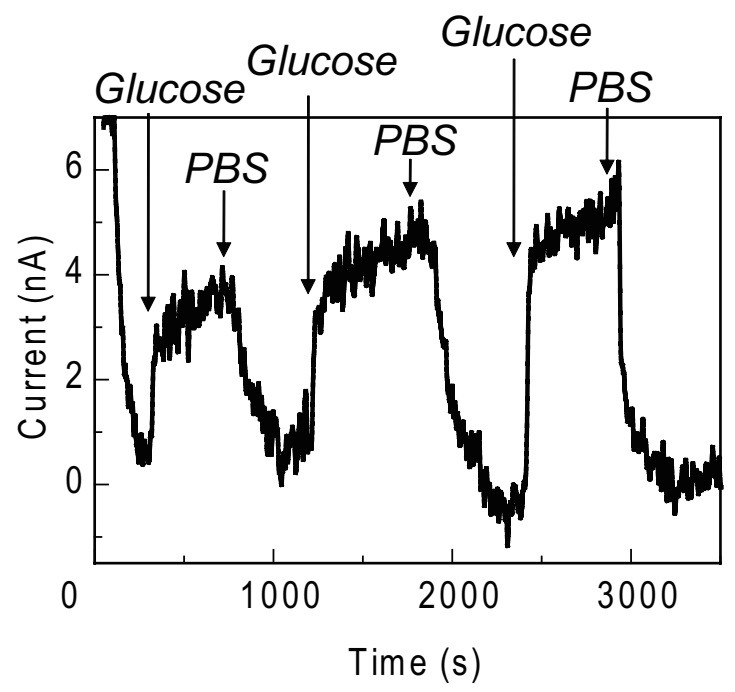

(b)

Fig. 5. (a) Schematic illustration of detection of glucose molecules. Glucose oxidase was covalently immobilized on the CNTs using linkers. (b) Time dependence of electrochemical signals determined by $\mathrm{CA}$ at an applied potential of $450 \mathrm{mV}$ vs $\mathrm{Ag} / \mathrm{AgCl}$. Glucose samples at 5, 10, and $20 \mathrm{mg} / \mathrm{mL}$ in PBS and PBS were alternately introduced into CNT electrodes from different inlets using two pumps. 


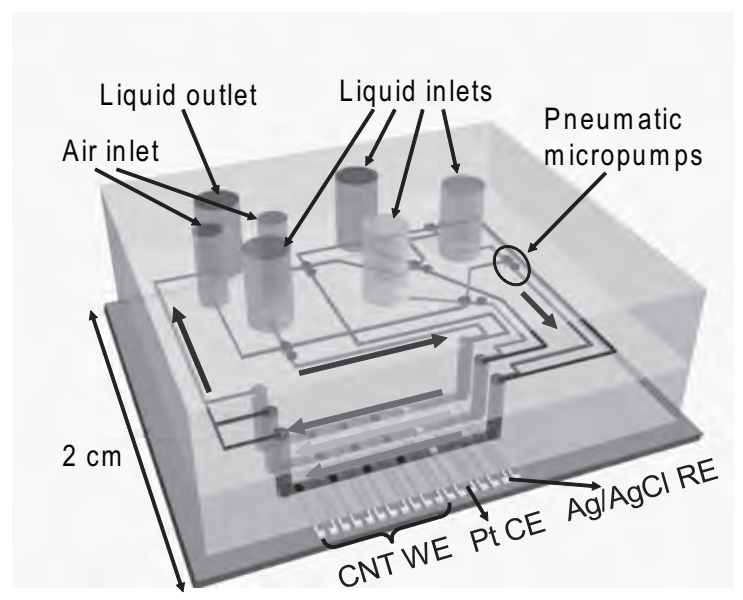

(a)

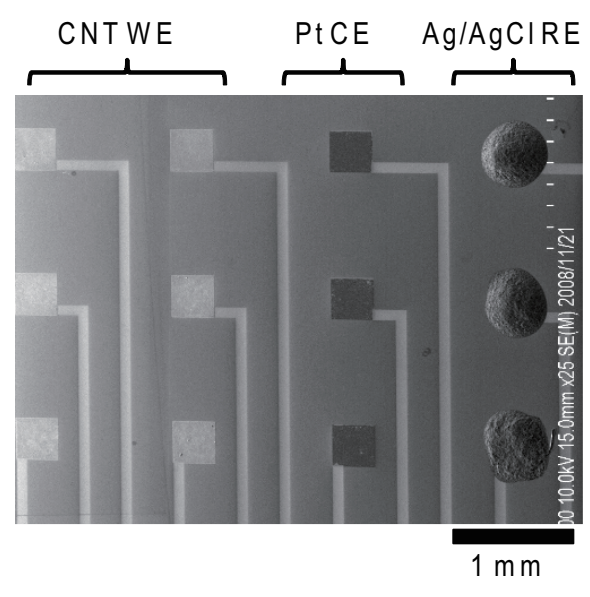

(b)

Fig. 6. (a) Schematic illustration of multibiosensors based on CNT electrodes. (b) SEM image of arrayed electrodes in microfluidic chip, which included twelve CNT, three Pt, and three $\mathrm{Ag} / \mathrm{AgCl}$ electrodes.

\section{Conclusions}

In this review, we provide an introduction to electrochemical amperometric biosensors based on CNT electrodes, which were directly synthesized on metal surfaces. The electrochemical measurements indicated that the CNT electrodes have a much higher sensitivity than conventional electrodes. Moreover, PSA in the range of $0.25-1 \mathrm{ng} / \mathrm{mL}$ can be effectively detected using the CNT electrodes, indicating that the label-free electrochemical biosensors are useful for the clinical diagnosis of prostate cancer. Microfluidic chips based on the CNT electrodes were also reviewed. The chip consisted of amperometric biosensors based on CNT-arrayed electrodes and microchannels with pneumatic micropumps. The reagents were introduced to arrayed electrodes without unexpected reverse flow and diffusion, and were automatically exchanged on the CNT electrodes. In this system, two types of cancer marker, PSA and hCG, were simultaneously detected by DPV. It is concluded that microfluidic chips with CNT-arrayed electrodes are promising candidates for the development of hand-held electrochemical multiplex biosensors.

\section{Acknowledgments}

We are grateful to Prof. E. Tamiya, Prof. Y. Takamura, and Dr. M. Chikae for their support in preparing the manuscript. The authors acknowledge the generous support from the Core Research for Evolutional Science and Technology, the Japan Science 
and Technology Corporation, the New Energy and Industrial Technology Development Organization, "Special Coordination Funds for Promoting Science and Technology: Yuragi Project" of the Ministry of Education, Culture, Sports, Science and Technology, Scientific Research from the Japan Society for the Promotion of Science, and Special Education, and Research Expenses (Post-Silicon Materials and Devices Research Alliance) from the Ministry of Education, Culture, Sports, Science and Technology of Japan.

\section{References}

1 M. J. P. Leiner: Anal. Chim. Acta 255 (1991) 209.

2 K. M. Muller, K. M. Arndt, K. Bauer and A. Pluckthun: Anal. Biochem. 259 (1998) 54.

3 L. A. Lyon, M. D. Musick and M. J. Natan: Anal. Chem. 70 (1998) 5177.

4 T. Drummond, M. Hill and J. Barton: Nat. Biotechnol. 21 (2003) 1192.

5 N. R. Stradiotto, H. Yamanaka, M. Valnice and B. Zanomi: J. Braz. Chem. Soc. 14 (2003) 159.

6 M. Bockrath, D. H. Cobden, P. L. McEuen, N. G. Chopra, A. Zettl, A. Thess and R. E. Smalley: Science 275 (1997) 1922.

7 K. Maehashi, H. Ozaki, Y. Ohno, K. Inoue, K. Matsumoto, S. Seki and S. Tagawa: Appl. Phys. Lett. 90 (2007) 023103.

8 Y. Ohno, Y. Asai, K. Maehashi, K. Inoue and K. Matsumoto: Appl. Phys. Lett. 94 (2009) 053112.

9 S. Tans, A. Verschueren and C. Dekker: Nature 393 (1998) 49.

10 K. Maehashi, Y. Ohno, K. Inoue, K. Matsumoto, T. Niki and H. Matsumura: Appl. Phys. Lett. 92 (2008) 183111.

11 D. Kaminishi, H. Ozaki, Y. Ohno, K. Maehashi, K. Inoue, K. Matsumoto, Y. Seri, A. Masuda and H. Matsumura: Appl. Phys. Lett. 86 (2005) 113115.

12 K. Maehashi, K. Matsumoto, K. Karman, Y. Takamura and E. Tamiya: Jpn. J. Appl. Phys. 43 (2004) L1558.

13 A. Star, J. Gabriel, K. Bradley and G. Gruner: Nano Lett. 3 (2003) 459.

14 K. Besteman, J. Lee, F. Wiertz, H. Heering and C. Dekker: Nano Lett. 3 (2003) 727.

15 K. Maehashi, T. Katsura, K. Karman, K. Matsumoto and E. Tamiya: Anal. Chem. 79 (2007) 782.

16 T. Katsura, Y. Yamamoto, K. Maehashi, Y. Ohno and K. Matsumoto: Jpn. J. Appl. Phys. 47 (2008) 2060.

17 Y. Yamamoto, Y. Ohno, K. Maehashi and K. Matsumoto: Jpn. J. Appl. Phys. 48 (2009) 06FJ01.

18 K. Maehashi, K. Matsumoto, Y. Takamura and E. Tamiya: Electroanalysis 21 (2009) 1285.

19 J. Wang: Electroanalysis 17 (2004) 7.

20 G. Liu, S. L. Riechers, M. C. Mellen and Y. Lin: Electrochem. Commun. 7 (2005) 1163.

21 S. Fei, J. Chen, S. Yao, G. Deng, D. He and Y. Kuang: Anal. Biochem. 339 (2005) 29.

22 N. S. Lawrence and J. Wang: Electrochem. Commun. 8 (2006) 71.

23 K. Maehashi, Y. Ohno, K. Inoue and K. Matsumoto: Appl. Phys. Lett. 85 (2004) 858.

24 J. Okuno, K. Maehashi, K. Matsumoto, K. Kerman, Y. Takamura and E. Tamiya: Electrochem. Commun. 9 (2007) 13.

25 L. Moreno, A. Merkoci, S. Alegret, S. H. Cassou and J. Saurina: Anal. Chim. Acta 507 (2004) 247. 
26 J. Okuno, K. Maehashi, K. Kerman, K. Matsumoto, Y. Takamura and E. Tamiya: Biosens. Bioelectron. 22 (2007) 2377.

27 A. Manz, N. Graber and H. M. Widmer: Sens. Actuators, B 1 (1990) 244.

28 D. J. Harrison, K. Fluri, K. Seiler, Z. Fan, C. S. Effenhauser and A. Manz: Science 261 (1993) 895.

29 D. R. Reyes, D. Iossifidis, P. Auroux and A. Manz: Anal. Chem. 74 (2002) 2623.

30 Y. Tsujita, K. Maehashi, K. Matsumoto, M. Chikae, S. Torai, Y. Takamura and E. Tamiya: Jpn. J. Appl. Phys. 47 (2008) 2064.

31 Y. Tsujita, K. Maehashi, K. Matsumoto, M. Chikae, Y. Takamura and E. Tamiya: Jpn. J. Appl. Phys. 48 (2009) 06FJ02. 